Article

\title{
Insulin-Like Peptide and FoxO Mediate the Trehalose Catabolism Enhancement during the Diapause Termination Period in the Chinese Oak Silkworm (Antheraea pernyi)
}

\author{
Ya-Na Li ${ }^{1}$, Xiao-Bing Ren ${ }^{2}$, Zhi-Chao Liu ${ }^{2}$, Bo Ye ${ }^{3}$, Zhen-Jun Zhao ${ }^{3}$, Qi Fan ${ }^{3}$, Yu-Bo Liu ${ }^{2}$, Jia-Ning Zhang ${ }^{2}$ \\ and Wen-Li Li ${ }^{2, *(D)}$ \\ 1 School of Bioengineering, Dalian University of Technology, Dalian 116024, China; liyana90@mail.dlut.edu.cn \\ 2 School of Life and Pharmaceutical Sciences, Dalian University of Technology, Panjin 124211, China; \\ rxb@mail.dlut.edu.cn (X.-B.R.); zhichaoliu@mail.dlut.edu.cn (Z.-C.L.); liuyubo@dlut.edu.cn (Y.-B.L.); \\ jnzhang@dlut.edu.cn (J.-N.Z.) \\ 3 Liaoning Ocean and Fisheries Science Research Institute, Liaoning Academy of Agricultural Sciences, \\ Dalian 116023, China; keyasyie@hotmail.com (B.Y.); zhenzhen1029@126.com (Z.-J.Z.); \\ qifan10001@163.com (Q.F.) \\ * Correspondence: biolwl@dlut.edu.cn
}

check for

updates

Citation: Li, Y.-N.; Ren, X.-B.; Liu, Z.-C.; Ye, B.; Zhao, Z.-J.; Fan, Q.; Liu, Y.-B.; Zhang, J.-N.; Li, W.-L. Insulin-Like Peptide and FoxO Mediate the Trehalose Catabolism Enhancement during the Diapause Termination Period in the Chinese Oak Silkworm (Antheraea pernyi). Insects 2021, 12, 784. https:// doi.org/10.3390/insects12090784

Academic Editor: Peter Armbruster

Received: 22 July 2021

Accepted: 28 August 2021

Published: 1 September 2021

Publisher's Note: MDPI stays neutral with regard to jurisdictional claims in published maps and institutional affiliations.

Copyright: (C) 2021 by the authors Licensee MDPI, Basel, Switzerland. This article is an open access article distributed under the terms and conditions of the Creative Commons Attribution (CC BY) license (https:/ / creativecommons.org/licenses/by/ $4.0 /)$.
Simple Summary: In insects, the insulin/insulin-like growth factor signalling (IIS) pathway regulates the carbohydrate and lipid metabolisms, and plays important roles in diapause regulation. Trehalose accumulates in many diapausing insects, as it is a major carbohydrate reserve and a stress protectant. Because of metabolism depression, the trehalose concentration is maintained at relatively high levels over the diapause phase. In the present study, bovine insulin injection triggered diapause termination and synchronous eclosion in Antheraea pernyi pupae. Moreover, treatment with bovine insulin elevated the trehalose catabolism in diapausing pupae. As a homologue of vertebrate insulin, insulinlike peptide $(A p I L P)$ enhances the trehalose catabolism during the diapause termination process. The transcription factor forkhead box $\mathrm{O}(A p F o x O)$ - the downstream target of the IIS pathway-exhibited a contrasting effect on the trehalose catabolism to that of ApILP. These results suggest that ApILP and $A p F o x O$ are involved in the regulation of trehalose catabolism during diapause termination in A. pernyi pupae.

Abstract: In insects, trehalose accumulation is associated with the insulin/insulin-like growth factor signalling (IIS) pathway. However, whether insulin-like peptide is involved in the regulation of the trehalose metabolism during diapause termination remains largely unknown. This study assessed whether insulin-like peptide (ApILP) enhances the trehalose catabolism in the pupae of Antheraea pernyi during their diapause termination process. Injection of $10 \mu \mathrm{g}$ of bovine insulin triggered diapause termination and synchronous adult eclosion in diapausing pupae. Moreover, treatment with bovine insulin increased the expression of trehalase 1A (ApTre-1A) and trehalase 2 (ApTre-2), as well as the activity of soluble and membrane-bound trehalase, resulting in a decline in trehalose levels in the haemolymph. Silencing ApILP via RNA interference significantly suppressed the expression of ApTre-1A and ApTre-2, thus leading to an increase in the trehalose concentration during diapause termination. However, neither injection with bovine insulin nor ApILP knockdown directly affected trehalase 1B (ApTre-1B) expression. Moreover, overexpression of the transcription factor forkhead box $\mathrm{O}(\mathrm{ApFoxO})$ induced an increase in trehalose levels during diapause termination; however, depletion of $A p F o x O$ accelerated the breakdown of trehalose in diapausing pupae by increasing the expression of ApTre-1A and ApTre-2. The results of this study help to understand the contributions of $A p I L P$ and $A p F o x O$ to the trehalose metabolism during diapause termination.

Keywords: Antheraea pernyi; RNA interference; overexpression; expression profile; trehalose metabolism 


\section{Introduction}

To ensure their survival, insects have developed a range of specific abilities to cope with extreme environments. In response to increasing environmental adversity, many insects switch their direct developmental program to diapause [1]. Diapause is a physiological state of developmental arrest, characterized by a depression of the metabolism, which allows individuals to survive under severe conditions of environmental stress [2-4]. The management and utilisation of stored energy play important roles in diapausing insects. Energetic reserves accumulate in diapause-destined individuals during the pre-diapause phase $[5,6]$. To enhance stress resistance, the main energy reserves (which include trehalose, glycogen, and lipids) are consumed in an economical manner because of metabolic depression throughout the diapause stage. In this way, insects can retain sufficient energy reserves to support themselves over an extended diapause period. These reserves have a profound effect on their development following diapause termination [7]. A recent study showed that metabolism enhances in response to diapause transition, suggesting that the change in metabolism may affect the progress of diapause [8].

The Chinese oak silkworm, Antheraea pernyi, is a traditional insect resource in China, which is highly valued for its silk as well as its rich nutritional properties [9]. When $A$. pernyi larvae perceive unfavourable environmental signals, such as short daylengths or low temperatures, they are programmed to enter a stage of facultative diapause during their pupal development [10]. As trehalose is a major circulating sugar, and also serves as an agent protecting individuals against environmental stress, its concentration is closely related to the occurrence, maintenance, and termination of diapause in many insect species [11-15]. Previous studies have reported that trehalose accumulates in diapause-destined A. pernyi pupae, and relatively high trehalose levels are maintained throughout the diapause phase; however, these levels decrease significantly after diapause termination, suggesting close regulation of the level of trehalose from the onset to the termination of diapause [16,17].

Insulin-like peptide (ILP) - a homologue of insulin—is a crucial controller of the consumption of carbohydrate reserves in insects. ILP has been reported to be involved in regulating haemolymph trehalose levels in various insects. Injection of bombyxin-the first insect ILP that was discovered-decreases the trehalose concentration by elevating trehalase activity in neck-ligated larvae of Bombyx mori [18]. Ablation of median neurosecretory cells that produce ILP in the insect brain causes elevated trehalose and glucose levels in the haemolymph of Drosophila melanogaster [19]. Similarly, knockdown of insulin-receptor genes significantly increases haemolymph trehalose levels and decreases expression of trehalase in Maruca vitrata [20]. The transcription factor forkhead box O (FoxO) is a downstream target, negatively regulated by the insulin/insulin-like growth factor signalling (IIS) pathway, and involved in the regulation of diverse cellular functions, such as differentiation, metabolism, proliferation, and survival [21]. In Caenorhabditis elegans, FoxO increases trehalose synthesis by upregulating trehalose synthase expression, which promotes starvation resistance [22]. This evidence suggests that insulin signalling might contribute to the regulation of trehalose levels. Furthermore, a previous study reported that injection of bovine insulin successfully induces diapause termination in the pupae of Pieris brassicae [23]. Thus, it was hypothesized that the IIS pathway participates in the regulation of the trehalose metabolism during diapause termination in $A$. pernyi pupae.

In the present study, the genes encoding ApILP and $A p F o x O$ were cloned and identified, and their contributions to the trehalose catabolism were assessed in A. pernyi pupae during diapause termination. Injection of bovine insulin and RNA interference (RNAi) experiments targeting the ApILP gene showed that ApILP promotes trehalose hydrolysis by upregulating both $A p T r e-1 A$ and $A p T r e-2 ;$ moreover, bovine insulin injection also increases the activity of soluble and membrane-bound trehalase during diapause termination. In addition, the results of overexpressing and silencing $A p F o x O$ suggest a functional association between $A p F o x O$ and the trehalose level in the haemolymph. The present study contributes to a better understanding of the roles of ApILP and ApFoxO in regulating the trehalose metabolism during diapause termination in the pupae of $A$. pernyi; it lays a foundation for 
later research on the diapause termination mechanism of $A$. pernyi, and facilitates improved utilisation of $A$. pernyi resources.

\section{Materials and Methods}

\subsection{Insects}

The larvae and pupae of the bivoltine strain Qing No. 6 of A. pernyi were obtained from the Sericultural Research Institute of Liaoning Province (Fengcheng, China). Eight fifthinstar larvae aged 10 days were employed and various tissues were dissected, including fat bodies, hemocytes, silk glands, midgut, Malpighian tubules, and epidermis. The fourthand fifth-instar larvae, early pupae, 5-day-old and 10-day-old pupae, and 1-day-old adults were also collected. Dissected tissues were immediately frozen in liquid nitrogen and then stored at $-80^{\circ} \mathrm{C}$ until use. Diapausing pupae were placed under a short-day photoperiod of L:D 9:15 at $25^{\circ} \mathrm{C}$ for 2 weeks to ensure that they remained in the state of diapause until their use in experiments. To break diapause, pupae were exposed to a long-day photoperiod of L:D 17:7 at $25^{\circ} \mathrm{C}$. The diapause state was determined based on the transparency of the epicranium $[17,24]$.

\subsection{Total RNA Extraction and Cloning of Full-Length $c D N A$}

Total RNA was extracted from the fat bodies, haemocytes, silk glands, midgut, Malpighian tubules, and epidermis of fifth-instar larvae after these tissues were ground in liquid nitrogen using the RNAiso Plus reagent (Takara, Shiga, Japan), in accordance with the manufacturer's instructions. First-strand cDNA was synthesized using the PrimeScript $^{\mathrm{TM}}$ 1st strand cDNA Synthesis Kit (Takara, Shiga, Japan), in accordance with the manufacturer's instructions. To clone the ApILP gene, the primers ApILP-F and ApILP-R were used for amplification. The PCR reaction contained $0.3 \mu \mathrm{L}$ of TaKaRa Ex Taq, $5 \mu \mathrm{L}$ of $10 \times$ Ex Taq buffer, $4 \mu \mathrm{L}$ of dNTP mixture, $33.7 \mu \mathrm{L}$ of $\mathrm{dH}_{2} \mathrm{O}, 2 \mu \mathrm{L}$ of cDNA, and $2.5 \mu \mathrm{L}$ of each primer $(10 \mu \mathrm{M})$. The reaction procedure was as follows: 40 cycles of denaturation at $94{ }^{\circ} \mathrm{C}$ for $30 \mathrm{~s}$, annealing at $45^{\circ} \mathrm{C}$ for $30 \mathrm{~s}$, and elongation at $72{ }^{\circ} \mathrm{C}$ for $1 \mathrm{~min}$. To clone the ApFoxO gene, PCR was performed with the primers ApFoxO-F and ApFoxO-R. Amplification cycling parameters were 40 cycles at $95^{\circ} \mathrm{C}$ for $30 \mathrm{~s}, 58^{\circ} \mathrm{C}$ for $30 \mathrm{~s}$, and $72{ }^{\circ} \mathrm{C}$ for $2 \mathrm{~min}$, which was followed by an extension at $72{ }^{\circ} \mathrm{C}$ for $10 \mathrm{~min}$. The amplification products were analysed on $1 \%$ agarose gels stained with ethidium bromide. The amplification products were then purified using a DNA gel extraction kit (TransGen, Beijing, China), and were subsequently sequenced by Sangon Biotech (Sangon Biotech, Shanghai, China).

\subsection{Sequence Alignment and Phylogenetic Tree Construction}

For homologous alignment, the deduced amino acid sequence, encoded by ApILP or $A p F o x O$, was compared to those of other insect species, which were downloaded from the GenBank database. Amino acid sequence alignments were performed using Clustal $\mathrm{X}$ software (version 2.0). Analyses of the molecular mass (kDa) and isoelectric point $(\mathrm{pI})$ of proteins were carried out using the ExPASy online server (https:/ /www.expasy.org/; accessed on 17 October 2020). The signal peptide was predicted using the SignalP 5.0 server (http:/ / www.cbs.dtu.dk/services/SignalP/; accessed on 17 October 2020). The phylogenetic tree was constructed in MEGA (version 7) using the neighbour-joining method with 1000 bootstrap replicates.

\subsection{Administration of Bovine Insulin}

Bovine insulin (Sigma-Aldrich, St. Louis, MO, USA) was first dissolved in $1 \mathrm{M} \mathrm{HCl}$; then, the $\mathrm{pH}$ was adjusted to 3 using $0.5 \mathrm{M} \mathrm{NaOH}$; finally, the solution was diluted to the desired concentration using $100 \mathrm{mM}$ PBS buffer ( $\mathrm{pH}$ 7.4). Various amounts of bovine insulin $(0,1,5,10,20$, and $40 \mu \mathrm{g} / 10 \mu \mathrm{L})$ were injected into diapausing $A$. pernyi pupae using a Hamilton microsyringe. Equal volumes of PBS buffer were used as controls. All treated pupae were incubated at $25^{\circ} \mathrm{C}$ and L:D 9:15, and adult emergence was recorded 
in each group. The experiment was conducted three times, and each group contained 20 individuals.

As treatment with a dose of $10 \mu \mathrm{g}$ bovine insulin could prompt subsequent development in nearly all diapausing $A$. pernyi pupae (as mentioned above), $10 \mu \mathrm{g}(10 \mu \mathrm{L})$ of bovine insulin was injected, and its effect on the trehalose catabolism was assessed. Equal volumes of PBS buffer were used as controls. Treated A. pernyi pupae were collected, and their gene expression, trehalose and glucose levels, and trehalase activity were analysed at selected time points up to $18 \mathrm{~d}$ post-injection.

\subsection{RNA Interference Targeting ApILP, ApFoxO, and ApTres}

With the gene-specific primers dsApILP-F and dsApILP-R (Table S1), containing the T7 polymerase promoter sequence, a $327 \mathrm{bp}$ dsRNA fragment that specifically targets ApILP (dsApILP) was synthesised. The Promega T7 expression kit (Promega, Madison, WI, USA) was used for in vitro transcription, following the manufacturer's protocol. The final concentration of dsApILP was adjusted to $5 \mu \mathrm{g} / \mu \mathrm{L}$ with nuclease-free water. To perform RNAi, pupae were first placed under the long-day photoperiod conditions for $17 \mathrm{~d}$ to break the diapause; then, $50 \mu \mathrm{g}$ of dsApILP was injected into the abdomens of pupae to silence ApILP. Equal amounts of dsRNA targeting the enhanced green fluorescent protein gene (dsEGFP), derived from a $435 \mathrm{bp}$ fragment of the EGFP gene, were injected into pupae in the control group. In this experiment, each group contained eight individuals, and was prepared in triplicate. The weight of the $A$. pernyi pupae was $\sim 8 \mathrm{~g}$. All treated pupae were collected for further analysis at 24,36, 48, and $72 \mathrm{~h}$ after dsRNA injection.

The 435 bp dsRNA fragment targeting $A p F o x O$ was prepared using ds $A p F o x O-F$ and dsApFoxO-R (Table S1), following the same method used for the synthesis of dsApILP. Similar to the RNAi experiment that targeted ApILP, $50 \mu \mathrm{g}(10 \mu \mathrm{L})$ of dsApFoxO was injected into the abdomens of diapausing pupae, and equal amounts of dsEGFP were injected ( $\sim 8 \mathrm{~g}$ mean fresh body weight) into pupae in the control group. In this experiment, each group also contained eight individuals, and was prepared in triplicate. At $48 \mathrm{~h}$ post-injection, all treated pupae were collected for further analysis.

To generate specific dsRNA fragments targeting ApTres, the lengths of the homologous dsRNAs for ApTre-1A, ApTre-1B, and ApTre-2 used in this study were 762, 779, and $773 \mathrm{bp}$, respectively. Three pairs of primers (Table S1) with the T7 polymerase promoter sequence were designed according to these regions. The dsRNA fragments targeting dsApTres were synthesized in vitro using the Promega T7 expression kit. For RNA interference, pupae were first placed under the long-day photoperiod conditions for $17 \mathrm{~d}$ to break diapause; then, $50 \mu \mathrm{g}(10 \mu \mathrm{L})$ each of dsApTre-1A, dsApTre-1B, and dsApTre-2 were injected into the abdomens of the pupae. Equal amounts of dsEGFP were injected (approximately $8 \mathrm{~g}$ mean fresh body weight) into pupae in the control group. In this experiment, each group also contained eight individuals, and was prepared in triplicate. At $48 \mathrm{~h}$ post-injection, all treated pupae were collected for further analysis.

\subsection{Baculovirus-Mediated Overexpression of ApFoxO in A. pernyi Pupae In Vivo}

The $A$. pernyi nucleopolyhedrovirus (ApNPV) expression vector system has been previously used for the stable expression of foreign genes in $A$. pernyi pupae $[25,26]$. To transiently overexpress $A p F o x O$ in vivo, the gene was cloned into the ApNPV transfer vector pApM748BE, and $1 \mu \mathrm{g}$ of pApM748BE/ApFoxO was co-transfected with $0.5 \mu \mathrm{g}$ of the genomic DNA of ApNPV- $\Delta p h / e g f p^{+}$into Tn-Hi5 cells $\left(1 \times 10^{6}\right)$ using Cellfectin II. After incubation at $27^{\circ} \mathrm{C}$ for $5 \mathrm{~d}$, the supernatant of the cell culture was harvested, and then $100 \mu \mathrm{L}$ of the supernatant was injected into $A$. pernyi pupae to generate the recombinant virus ApNPV- $\Delta p h / \triangle e g f p / A p F o x O^{+}$. Modified plaque purification was used to isolate the recombinant virus. Selected cells containing the recombinant virus were picked up using sterile Pasteur pipettes, resuspended in $100 \mu \mathrm{L}$ of TNM-FH serum-free medium, and then the viral supernatant was injected into A. pernyi pupae. After incubation at $25^{\circ} \mathrm{C}$ for $7 \mathrm{~d}$, the tissue (including haemolymph and fat bodies of the infected pupae) was used to extract 
viral DNA using GenElute ${ }^{\mathrm{TM}}$ Mammalian Genomic DNA Miniprep Kits (Sigma-Aldrich, St. Louis, MO, USA). The purity of the recombinant virus was assessed by PCR with Pap-seqF and Pap-seqR primers. The titre of the recombinant virus in the haemolymph of infected pupae was estimated according to the method of Reed and Muench [27]. The supernatant of the haemolymph of infected pupae was diluted 10 times with TNM-FH serum-free medium, filter-sterilized through a $0.22 \mu \mathrm{m}$ filter (Millipore), and then injected into pupae $\left(10^{5} \mathrm{pfu} / \mathrm{pupa}\right)$, which had been subjected to the long-day photoperiod for 14 days. Infected pupae were incubated at $25^{\circ} \mathrm{C}$ and were collected for further bioassays $6 \mathrm{~d}$ after injection of the recombinant virus ApNPV- $\Delta p h / \triangle e g f p / A p F o x O^{+}$. Pupae injected with ApNPV- $\Delta p h / e g f p^{+}$served as controls.

\subsection{Quantitative Real-Time PCR}

Quantitative real-time PCR (qRT-PCR) was conducted using a LightCycler ${ }^{\circledR} 96$ (Roche, Basel, Switzerland) with SYBR ${ }^{\circledR}$ Premix Ex Taq ${ }^{\mathrm{TM}}$ II (Takara, Shiga, Japan). The following conditions were applied: pre-incubation at $95^{\circ} \mathrm{C}$ for $30 \mathrm{~s}$, followed by 40 cycles of denaturation at $95^{\circ} \mathrm{C}$ for $5 \mathrm{~s}$, and annealing and elongation at $60^{\circ} \mathrm{C}$ for $30 \mathrm{~s}$. All gene-specific primers used for qRT-PCR are listed in Table S1. The relative expression levels of target genes were normalised with the two stable reference genes actin and RP49, and expression levels were calculated using the $2^{-\Delta \Delta c t}$ method [28].

\subsection{Measurement of Trehalose and Glucose Levels}

The haemolymph of A. pernyi pupae was collected, and trehalose and glucose were extracted. The extraction was performed following a previously described method [17,29]. Extracted samples were analysed with an Agilent HPLC system equipped with an evaporative light-scattering detector and a sugar-D, $\mathrm{NH}_{2}$-MS packed column $(4.6 \mathrm{~mm}$ I.D. $\times 250 \mathrm{~mm}$, COSMOSIL, Nacalai Tesque, Kyoto, Japan). The mobile phase consisted of a mixture of acetonitrile and water $(80: 20 \mathrm{v} / \mathrm{v})$, and the flow rate was $1 \mathrm{~mL} / \mathrm{min}$. The quantities of trehalose and glucose present in the samples were calculated according to standard solutions of trehalose and glucose, respectively.

\subsection{Measurement of Trehalase Activity}

The activity of soluble and membrane-bound trehalase was determined according to a previously described procedure [11,30]. Briefly, after removal of the puparium, the whole bodies of $A$. pernyi pupae were ground in $20 \mathrm{mM}$ PB buffer, sonicated for $30 \mathrm{~s}$, and centrifuged at $4{ }^{\circ} \mathrm{C}$ and $12,000 \times g$ for $30 \mathrm{~min}$. The supernatant was directly used to measure the activity of soluble trehalase, and the precipitate was used for a membranebound trehalase activity assay after resuspension in $20 \mathrm{mM}$ PB buffer; then, $200 \mu \mathrm{L}$ of soluble or membrane-bound trehalase fraction extract was mixed with $250 \mu \mathrm{L}$ of $40 \mathrm{mM}$ trehalose and $550 \mu \mathrm{L}$ of $20 \mathrm{mM} \mathrm{PB}$ buffer. The reaction mixture was incubated at $25^{\circ} \mathrm{C}$ for $30 \mathrm{~min}$, and then in boiling water for $5 \mathrm{~min}$. After centrifugation, the supernatant was used to measure the glucose content with a Glucose Assay Kit (Comin, Suzhou, China).

\subsection{Statistical Analysis}

Three biological and three technical replicates were used for each experiment. All data are presented as means \pm standard errors, determined using SPSS software version 22.0 (SPSS Inc., Chicago, IL, USA). Differences between groups were determined using Student's $t$-tests (for comparisons between two mean values) or by one-way analysis of variance (ANOVA) followed by a least significant difference (LSD) test (for comparisons between multiple mean values). Differences at $p<0.05$ were considered significant.

\section{Results}

\subsection{Cloning and Expression Profiles of ApILP and ApFoxO}

Based on our previous transcriptome data on $A$. pernyi (unpublished), the full-length cDNAs of ApILP and ApFoxO were obtained. The ApILP sequence consists of $327 \mathrm{bp}$ and 
encodes a polypeptide of 108 amino acids, showing homology (20-38\%) to other insect ILPs (Figures S1A and S2A). The open reading frame of ApFoxO consists of $1563 \mathrm{bp}$, and was translated into a protein of 520 amino acids. BLAST analysis showed that the deduced amino acid sequence of $A p F o x O$ has high homology (81-89\%) with other insects' FoxOs (Figures S1B and S2B).

The expression patterns of both genes were analysed from different tissues of fifthinstar larvae and at their different developmental stages. The tissue expression data show that $A p I L P$ and $A p F o x O$ expression were detected in all tested tissues; however, the expression levels of $A p I L P$ and $A p F o x O$ were significantly higher in the fat body than in other tissues (Figure 1A,D). The transcripts of ApILP and ApFoxO were also expressed at all developmental stages of $A$. pernyi. ApILP expression was significantly higher in the adult stage compared with other stages, while $A p F o x O$ exhibited significantly higher expression levels at the larval stage (Figure 1B,E). In addition, ApILP expression increased significantly on days 20 and 25 after long-day photoperiod treatment to break diapause. However, the expression of $A p F o x O$ followed an up-down-up pattern during the long-day photoperiod treatment period, suggesting that ApILP and ApFoxO might be involved in the regulation of the developmental process at diapause termination and post-termination phases (Figure 1C,F).

A
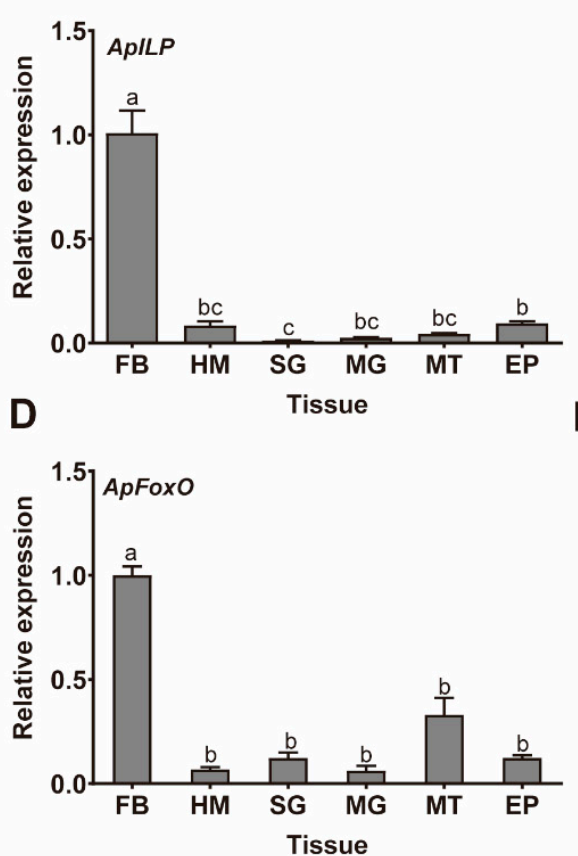

B
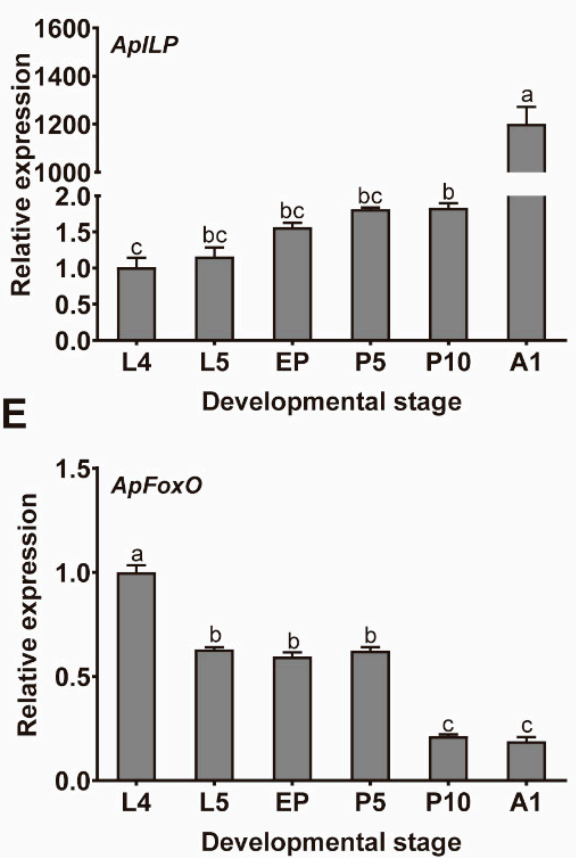

C
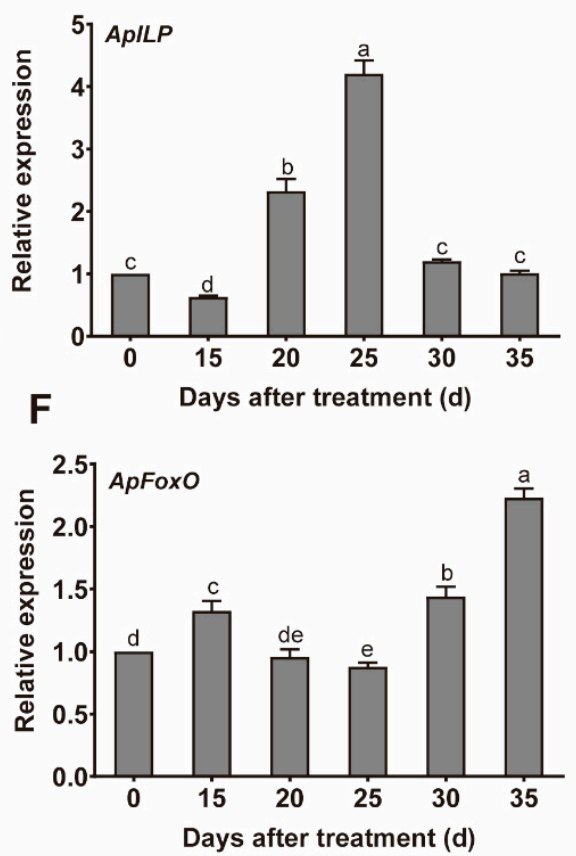

Figure 1. Temporal and spatial expression patterns of $A p I L P$ and $A p F o x O$ in Antheraea pernyi. (A,D) Relative expression levels of $A p I L P$ and $A p F o x O$, respectively, in different tissues of fifth-instar larvae; abbreviations: fat body (FB); hemocyte (HM); silk gland (SG); midgut (MG); Malpighian tubules (MT); epidermis (EP). (B,E) Expression profiles of ApILP and $A p F o x O$, respectively, at different developmental stages; abbreviations: 4th- and 5th-instar larvae (L4 and L5, respectively); early pupae (EP); 5-day-old and 10-day old pupae (P5 and P10, respectively); 1-day-old adults (A1). (C,F) Changes in the relative expression levels of $A p I L P$ and $A p F o x O$, respectively, after diapausing pupae were subjected to long-day photoperiod (L:D 17:7) treatment to break diapause. Based on the transparency of the epicranium, treated pupae were collected for qRT-PCR analysis on indicated days $(15,20,25,30$, and $35 \mathrm{~d})$ after long-day photoperiod (L:D 17:7) treatment. Diapausing pupae (day 0 ) were used as a control group. The period of the long-day photoperiod treatment incorporated two phases: the diapause termination phase (which continued until day 20 of treatment), and the post-termination phase (which started on day 25 and ended on day 35 of treatment). The mRNA levels were normalised to the reference genes actin and RP49. Data represent the means of three independent experiments, and error bars are SEs. Different letters above the error bars indicate significant differences at $p<0.05$. 


\subsection{Bovine Insulin Induces Diapause Termination}

The insect IIS pathway is involved in the regulation of insect development, longevity, cell cycle, metabolism, and female reproduction [31]. Previous studies have reported that the repression of ILP is associated with the generation of a diapause phenotype, suggesting that ILP plays an important role in the regulation of diapause in insects [32-34]. Because bovine insulin has $21.24 \%$ amino acid sequence homology with ApILP, the effect of bovine insulin injection on breaking diapause was investigated (Figure 2A). At a bovine insulin injection dose of $1 \mu \mathrm{g}$, treatment failed to produce any clear result. With increasing dose, the eclosion rate was significantly stimulated, and the best emergence rate $(100 \%)$ was achieved at $10 \mu \mathrm{g}$. However, when the applied dose exceeded $10 \mu \mathrm{g}$, the emergence rate started to decline slightly ( $95 \%$ at $20 \mu \mathrm{g}$ and $85 \%$ at $40 \mu \mathrm{g}$ ). Incubation times varied from $44 \pm 4$ days $(40 \mu \mathrm{g})$ to $29 \pm 2$ days $(10 \mu \mathrm{g})$. These results show that bovine insulin injection can effectively promote diapause termination in A. pernyi pupae.

A

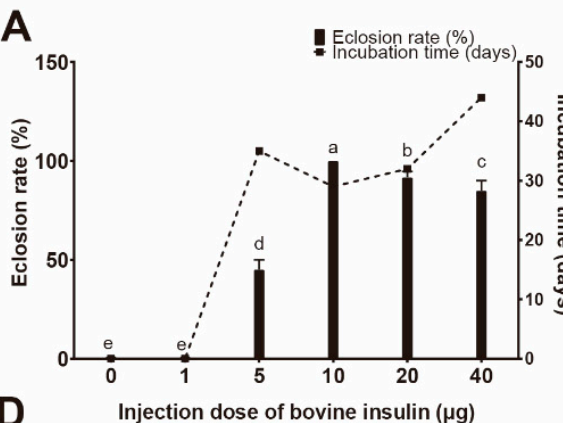

Injection dose of bovine insulin $(\mu \mathrm{g})$
Q Control
Bovine insulin

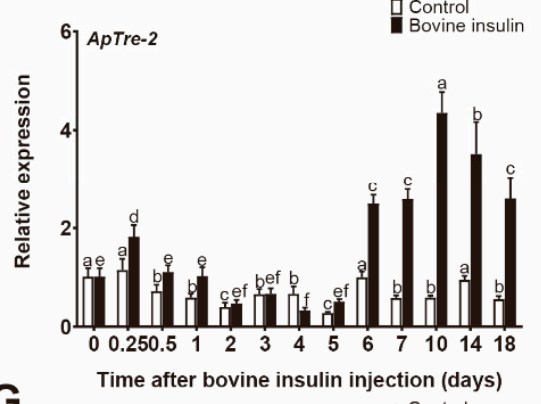

G

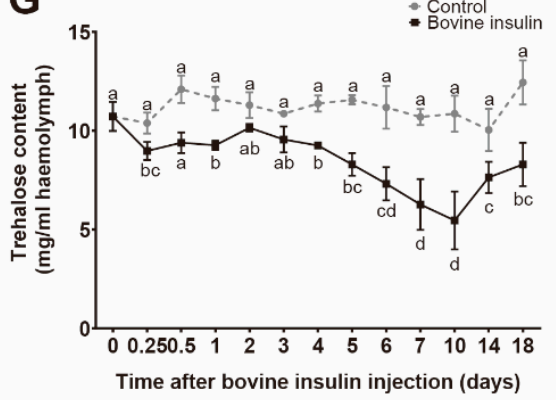

B
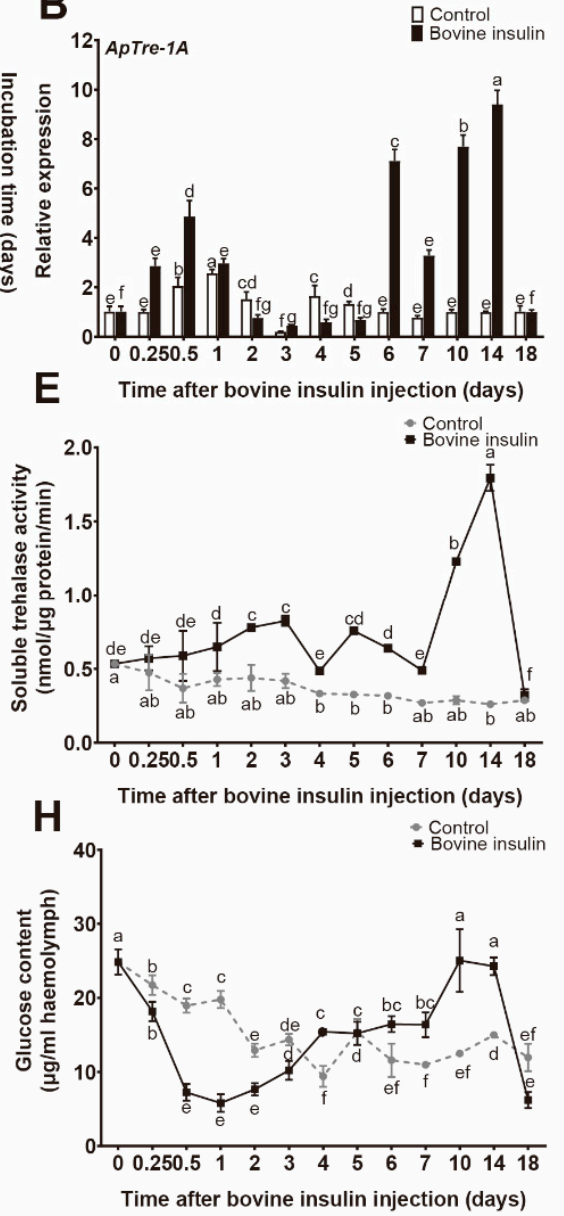

C
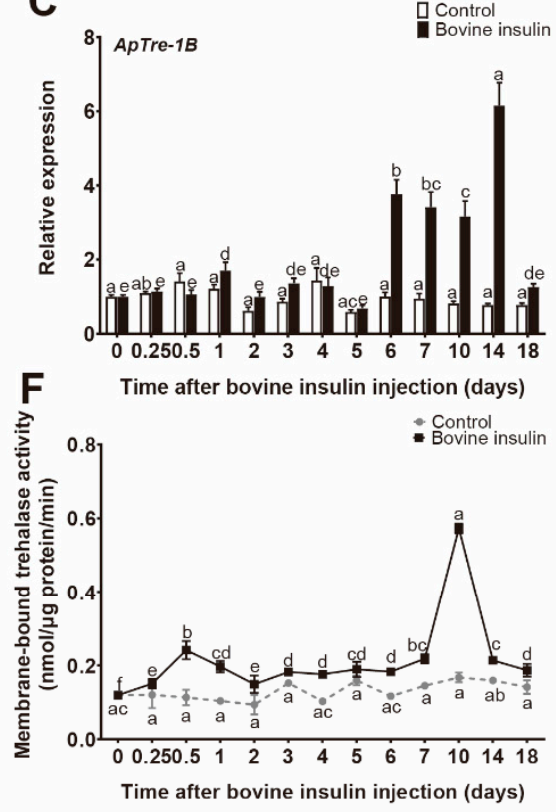

Figure 2. Bovine insulin injection induced diapause termination and enhanced trehalose catabolism in A. pernyi diapausing pupae. (A) Effect of different doses of bovine insulin on breaking the diapause of $A$. pernyi pupae. Diapausing pupae were exposed to a short-day photoperiod of L:D 9:15 at $25^{\circ} \mathrm{C}$ after bovine insulin injection. Eclosion rates were recorded. Each group consisted of 20 individuals, and error bars indicate the mean $\pm \mathrm{SE}$ of three replicates. (B-D) Relative expression levels of ApTre-1A, ApTre-1B, and ApTre-2, respectively, induced by bovine insulin injection. The mRNA levels were normalised to the reference genes actin and RP49, and pupae treated with PBS buffer served as controls (CK). The mRNA levels of ApTres in pupae before treatment $(0 \mathrm{~h})$ were defined as 1 . (E,F) Changes in soluble and membrane-bound trehalase activity, respectively, caused by bovine insulin injection. $(\mathbf{G}, \mathbf{H})$ Influence of bovine insulin treatment on haemolymph trehalose and glucose levels, respectively. The dotted lines represent the control group (which received PBS buffer), while the solid lines represent the bovine-insulin-treated group. Data are means of three independent experiments, and error bars are SEs. Different letters above the error bars indicate significant differences at $p<0.05$. 


\subsection{Bovine Insulin Enhances Trehalose Catabolism}

Given the effect bovine insulin has on the breaking of diapause in A. pernyi, the next test assessed whether trehalose catabolism was affected by bovine insulin. qRT-PCR analysis showed that bovine insulin injection triggered significant increases in ApTre- $1 A$, ApTre-1B, and ApTre-2 mRNA levels. Compared with the control group, injection of bovine insulin induced a rapid increase of ApTre-1A expression 6-24 h after injection; however, ApTre-1A expression decreased 2-5 d after treatment, and again dramatically increased from 6 to $14 \mathrm{~d}$ after bovine insulin injection, peaking at $14 \mathrm{~d}$ post-injection (where the level was 9.40-fold higher than that of the control group) (Figure 2B). Similarly, the first increase in ApTre-2 expression was observed 6-24 $\mathrm{h}$ after injection of bovine insulin; then, ApTre-2 expression was significantly upregulated from 6 to $18 \mathrm{~d}$ after bovine insulin treatment, and the mRNA level of ApTre-2 was 7.34-fold higher than that of the control group at $10 \mathrm{~d}$ post-injection (Figure 2D). In addition, the relative expression of ApTre-1B significantly increased on days $1,6,7,10,14$, and 18 after bovine insulin injection, in comparison with the control group (Figure 2C). Injection of bovine insulin significantly elevated the enzyme activity of soluble and membrane-bound trehalase in diapausing $A$. pernyi pupae, compared with pupae from the control group (which received PBS buffer only). Soluble trehalase activity gradually increased with bovine insulin injection from $6 \mathrm{~h}$ to $14 \mathrm{~d}$ post-injection, especially from $7 \mathrm{~d}$ to $14 \mathrm{~d}$, during which time a sharp increase was observed; however, the magnitude of the increase diminished clearly at $4 \mathrm{~d}$ and $7 \mathrm{~d}$ (Figure 2E). Similarly, the activity of membrane-bound trehalase increased after bovine insulin injection, especially at $12 \mathrm{~h}$ and at $10 \mathrm{~d}$ after treatment (Figure 2F).

In addition, injection of bovine insulin caused a significant decrease in trehalose concentration in diapausing A. pernyi pupae. The trehalose concentration of the control group remained relatively constant at $11.16 \pm 0.67 \mathrm{mg} / \mathrm{mL}$, while it decreased from $10.72 \mathrm{mg} / \mathrm{mL}$ $(0 \mathrm{~h})$ to $5.46 \mathrm{mg} / \mathrm{mL}(10 \mathrm{~d})$ following bovine insulin treatment (Figure $2 \mathrm{G}$ ). Moreover, the glucose content of pupae in the control group decreased slightly and fluctuated over a small range during the study period; however, the concentration of glucose in pupae treated with bovine insulin followed a down-up-down pattern, which is roughly the opposite trend compared with the trehalose content (Figure $2 \mathrm{H}$ ).

\subsection{ApILP RNAi Suppresses the Utilization of Trehalose}

Bovine insulin injection induces the breakdown of trehalose, suggesting that ApILP-a homologue of vertebrate insulin - might be involved in the modulation of haemolymph trehalose levels in A. pernyi. The ApILP gene was silenced by an RNAi experiment to further confirm its role in the regulation of the trehalose catabolism. After injection of ApILP-specific dsRNA, expression levels of ApILP were $21.4 \%, 71.2 \%, 50.0 \%$, and $49.1 \%$ lower than those of the control group at $24 \mathrm{~h}, 36 \mathrm{~h}, 48 \mathrm{~h}$, and $72 \mathrm{~h}$, respectively. This suggests that $d s A p I L P$ injection significantly suppressed the mRNA level of ApILP, and that the suppression lasted for at least $72 \mathrm{~h}$, leading to an effective RNAi response (Figure $3 \mathrm{~A}$ ). After injection of ApILP-specific dsRNA, the transcription levels of ApTre-1A and ApTre-2 decreased (Figure 3B,D), while the transcription level of ApTre-1B increased (Figure 3C). Under this RNAi condition, the trehalose levels in the haemolymph exceeded those of the control group (Figure $3 \mathrm{E}$ ). In contrast, ds ApILP treatment resulted in a significant reduction in the glucose content compared with the control group (Figure 3F). These results indicate that the suppression of ApILP expression inhibits trehalose utilisation. 
A
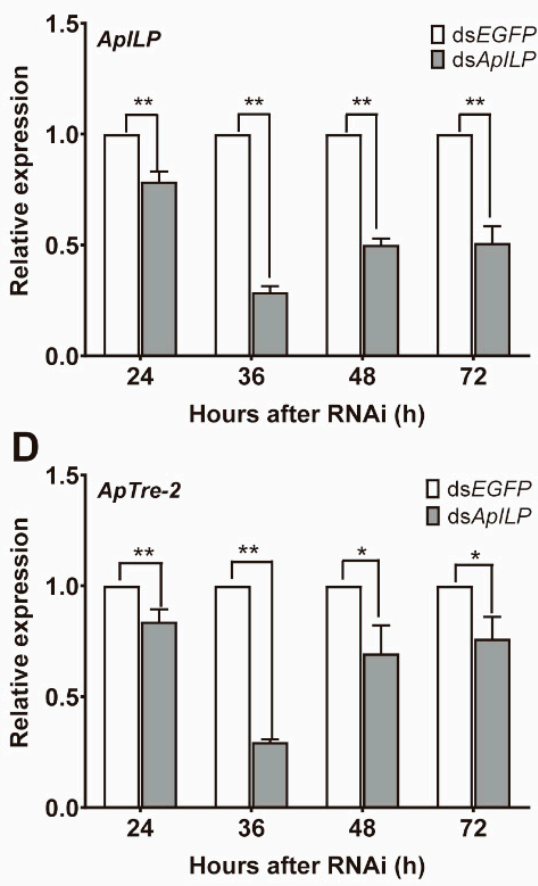

B
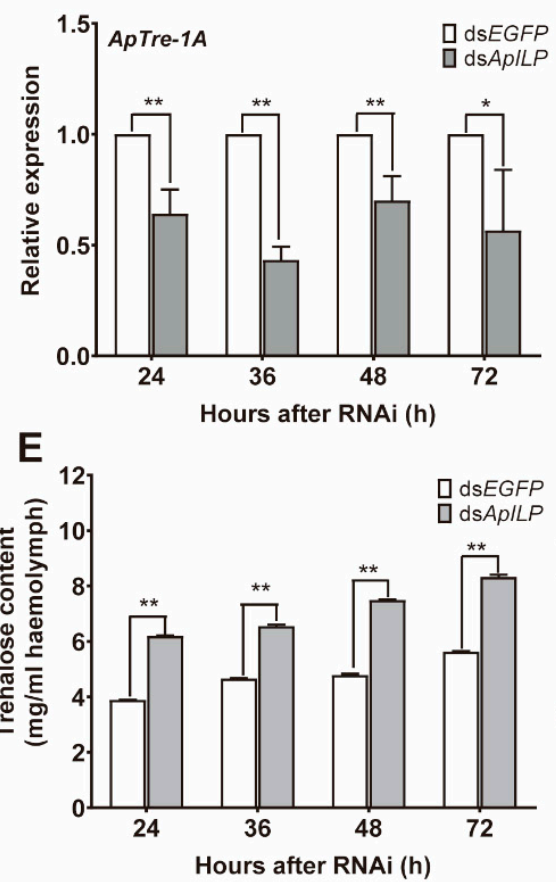

C

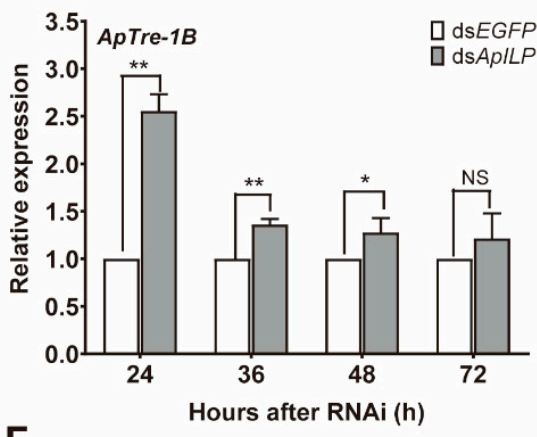

$\mathbf{F}$

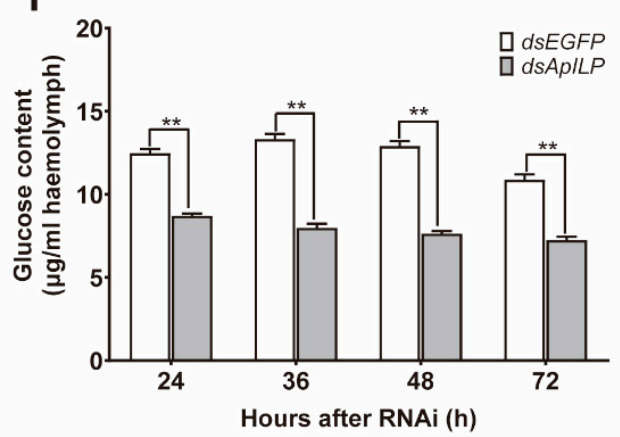

Figure 3. Effects of ApILP-specific RNA interference (RNAi) on trehalose metabolism in diapausing $A$. pernyi pupae during diapause termination. To effectively break diapause, diapausing $A$. pernyi pupae were placed under the long-day photoperiod conditions (L:D 17:7) for 17 days before receiving either dsApILP or dsEGFP treatments. (A-D) Relative expression levels of ApILP, ApTre-1A, ApTre-1B, and ApTre-2, respectively. (E,F) Changes in trehalose and glucose levels, respectively, in the haemolymph. mRNA levels were normalised to those of the reference genes actin and RP49, and the group injected with dsEGFP served as controls. Data are means of three independent experiments, and error bars are SEs. Single asterisks $(p<0.05)$ and double asterisks $(p<0.01)$ represent significant differences between the dsEGFP and dsApILP groups in the same time period.

\subsection{Effect of Overexpressing and Silencing ApFoxO on Trehalose Metabolism}

To further confirm that the IIS pathway is involved in the regulation of trehalose levels, the gene $A p F o x O$ - the downstream target of insulin signalling - was overexpressed and silenced. Compared with the injection of EGFP-baculovirus, injection of ApFoxO-baculovirus into A. pernyi pupae led to a dramatic increase in the $A p F o x O$ mRNA levels, and downregulated the mRNA levels of ApTre-1A and ApTre-2. However, ApTre-1B expression was slightly upregulated after overexpression of $A p F o x O$ (Figure 4A). Furthermore, a decrease in both soluble and membrane-bound trehalase activity was observed (Figure 4B), which resulted in an increased trehalose level and a decreased glucose level, when the gene of $A p F o x O$ was overexpressed (Figure $4 \mathrm{C}, \mathrm{D}$ ). In contrast, knockdown of $A p F o x O$ in diapausing pupae induced upregulation of $A p T r e-1 A$ and ApTre-2 expression, but slightly downregulated ApTre-1B expression compared with the control group (Figure 5A). In addition, increases in soluble and membrane-bound trehalase activity accelerated the conversion of trehalose into glucose when $A p F o x O$ expression was silenced (Figure 5B-D). These results suggest that $A p F o x O$ expression is positively correlated with trehalose levels by decreasing the mRNA levels of ApTre- $1 A$ and ApTre-2 and their corresponding activities. 
A

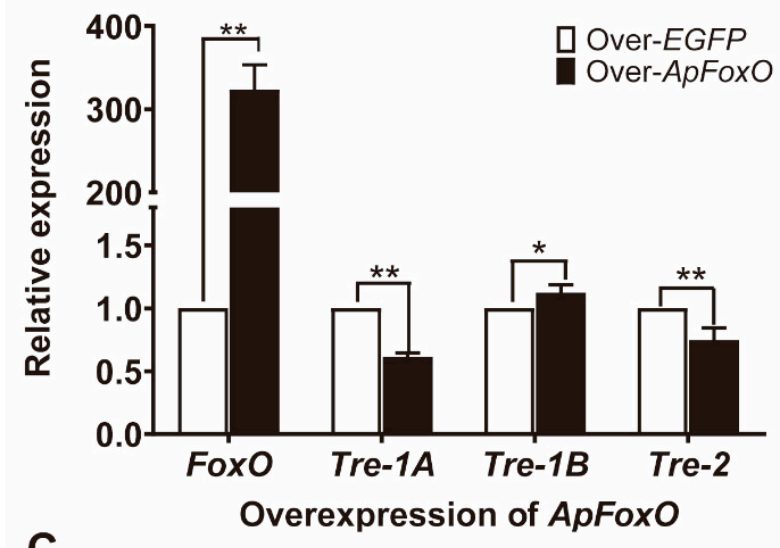

C

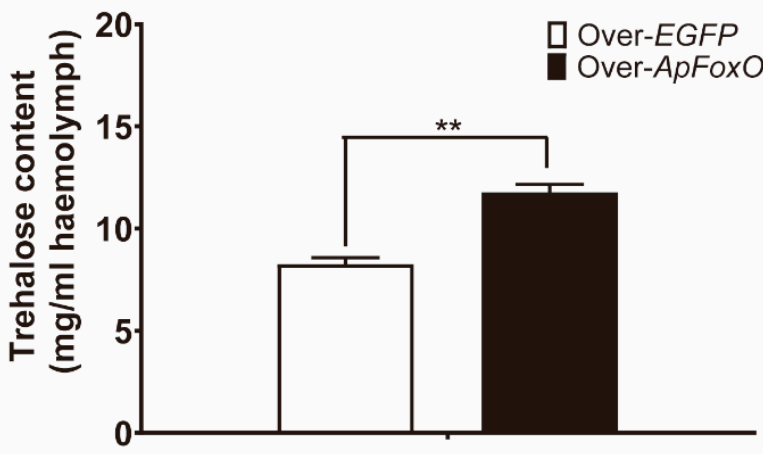

Overexpression of ApFoxO
B

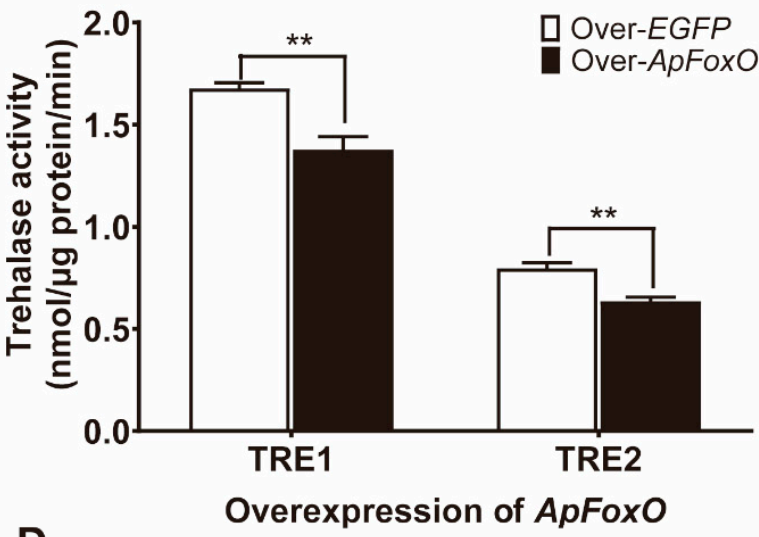

D

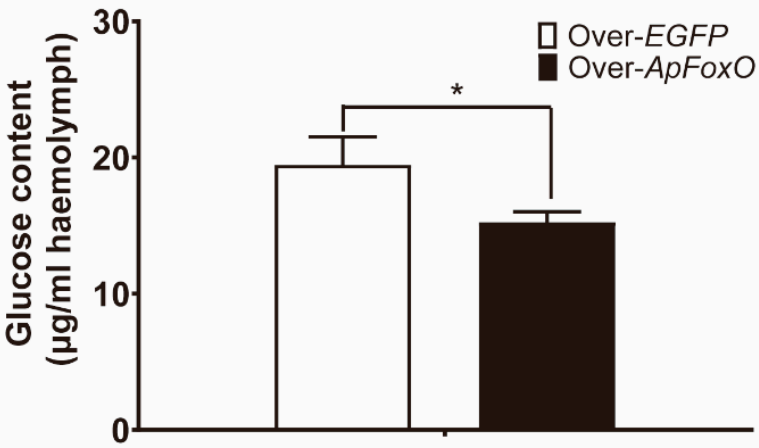

Overexpression of ApFoxO

Figure 4. Overexpression of $A p F o x O$ suppressed trehalose catabolism in A. pernyi pupae during diapause termination. All diapausing pupae were placed under the long-day photoperiod conditions (L:D 17:7) for 14 days to break diapause; then, they were injected with the recombinant virus ApNPV, containing either ApFoxO or EGFP cDNA under the promoter of the viral polyhedrin (polh) gene. Six days after the injection of baculovirus, pupae were collected for further analysis. (A) Relative expression levels of ApFoxO, ApTre-1A, ApTre-1B, and ApTre-2. (B) Changes in soluble (TRE1) and membranebound (TRE2) trehalase activity. (C,D) Changes in trehalose and glucose levels, respectively, in the haemolymph. mRNA levels were normalised to those of the reference genes actin and $R P 49$, and the group used for the overexpression of EGFP served as controls. Data are means of three independent experiments, and error bars are SEs. Single asterisks $(p<0.05)$ and double asterisks $(p<0.01)$ represent significant differences between the dsEGFP and dsApILP groups in the same time period.

\subsection{Knockdown of ApTres Suppresses Trehalose Catabolism}

To investigate the function of these ApTres genes and their relationship in the regulation of trehalose levels in the haemolymph, pupae were injected with specific dsRNAs targeting ApTre-1A, ApTre-1B, and ApTre-2. Pupae were collected for further analysis at $48 \mathrm{~h}$ after injection. The pupae injected with EGFP-specific dsRNA served as controls. Compared with the control group, the expression of ApTre-1A, ApTre-1B, and ApTre-2 decreased to $74.5 \%, 72.3 \%$, and $73.7 \%$, respectively, at $48 \mathrm{~h}$ after the injection of the corresponding dsRNA. This indicates that RNAi targeting each ApTre was successful in each group (Figure 6A-C). In addition to injection of the corresponding dsRNA, injection of ApTre-1A and ApTre$1 B$ dsRNAs also decreased ApTre-2 expression (Figure 6C). The expression of ApTre-1A and $A p T r e-1 B$ increased significantly after $A p T r e-2$ RNAi injection. In addition, $A p T r e-1 A$ showed a slight increase after $A p$ Tre-1B RNAi injection, while ApTre-1B also increased after ApTre-1A RNAi injection (Figure 6A,B). These changes in the expression levels of ApTres indicate possible inter-regulations between different ApTres. Silencing ApTre- $1 A$ and $A p T r e-1 B$ decreased the activity of both soluble and membrane-bound trehalase; however, silencing ApTre-2 decreased membrane-bound trehalase activity but increased soluble 
trehalase activity. These changes in trehalase activity were generally consistent with their corresponding expression level trends (Figure 6F,G). Moreover, knockdown of ApTre- $1 A$, ApTre-1B, or ApTre-2 resulted in an obvious increase in trehalose levels in the haemolymph, but caused a reduction in haemolymph glucose levels, compared with dsEGFP treatment (Figure 6D,E).

A
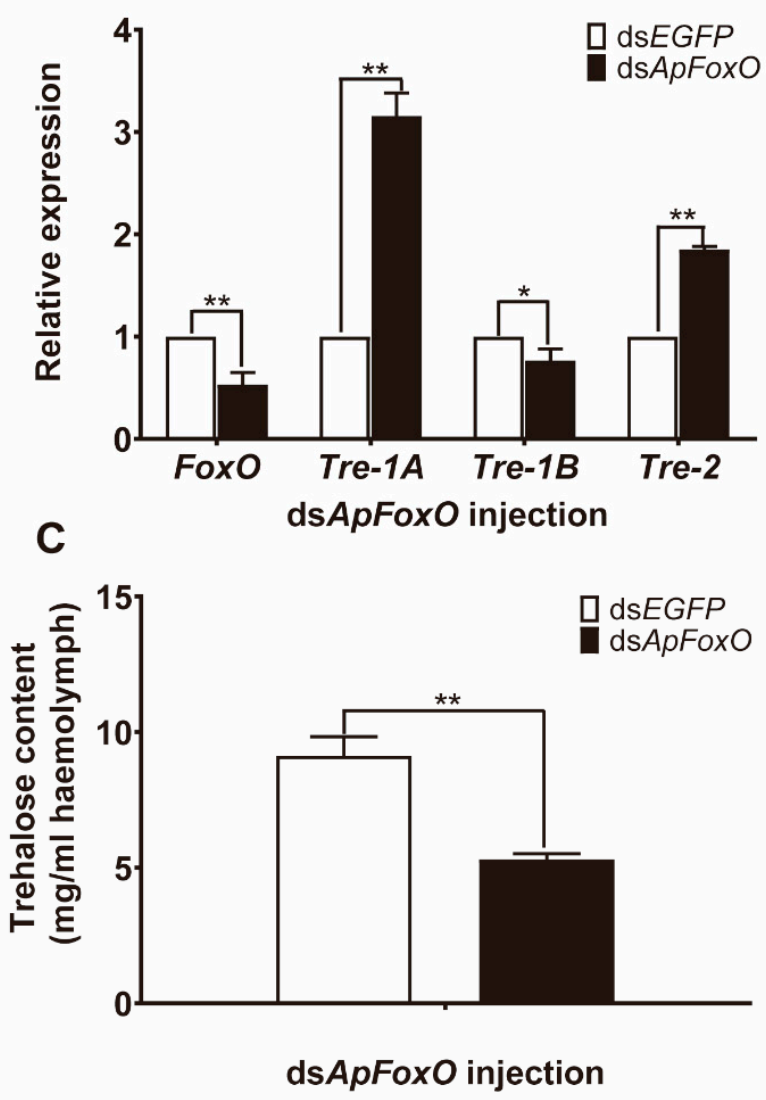

B
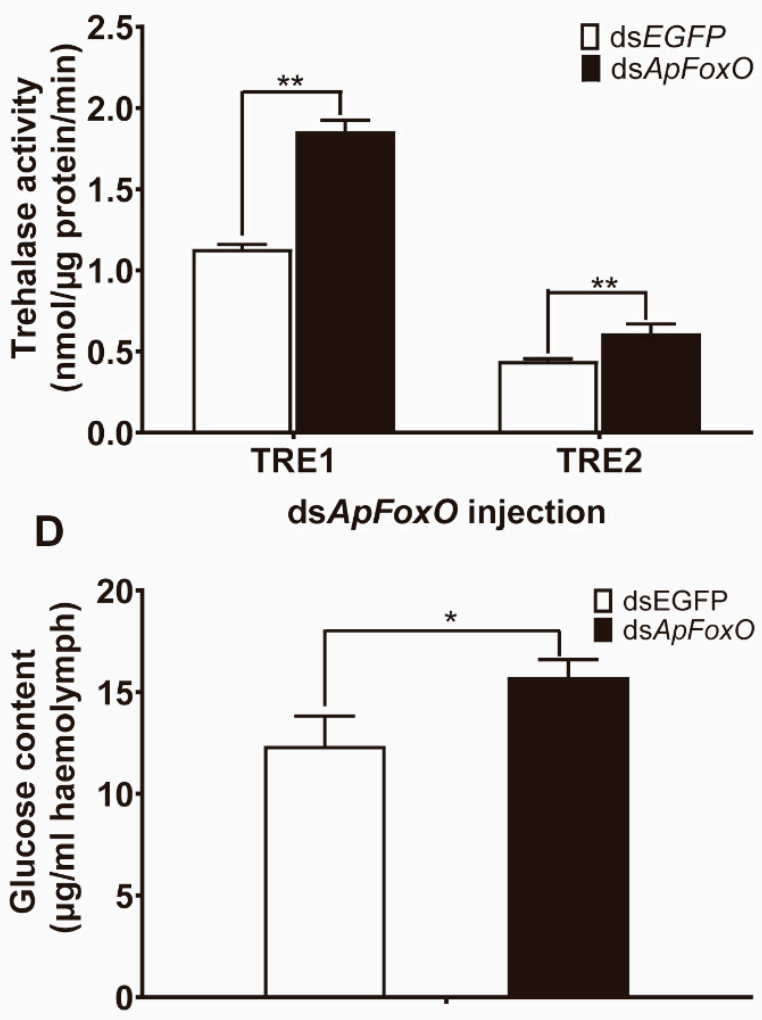

dsApFoxO injection

Figure 5. Effects of $A p F o x O$-specific RNAi on trehalose metabolism in diapausing A. pernyi pupae. (A) Relative expression levels of $A p F o x O$, ApTre-1A, ApTre-1B, and ApTre-2 at $48 \mathrm{~h}$ after dsApFoxO injection in comparison to controls (dsEGFP injection). (B) Changes in soluble (TRE1) and membrane-bound (TRE2) trehalase activity caused by ds $A p F o x O$ or dsEGFP treatment at $48 \mathrm{~h}$ post-injection. (C,D) Changes in trehalose and glucose content, respectively, in the haemolymph at $48 \mathrm{~h}$ post-injection. mRNA levels were normalised to those of the reference genes actin and $R P 49$, and the group injected with dsEGFP served as controls. Data are means of three independent experiments, and error bars are SEs. Single asterisks $(p<0.05)$ and double asterisks $(p<0.01)$ represent significant differences between the dsEGFP and dsApFoxO groups in the same time period. 
A
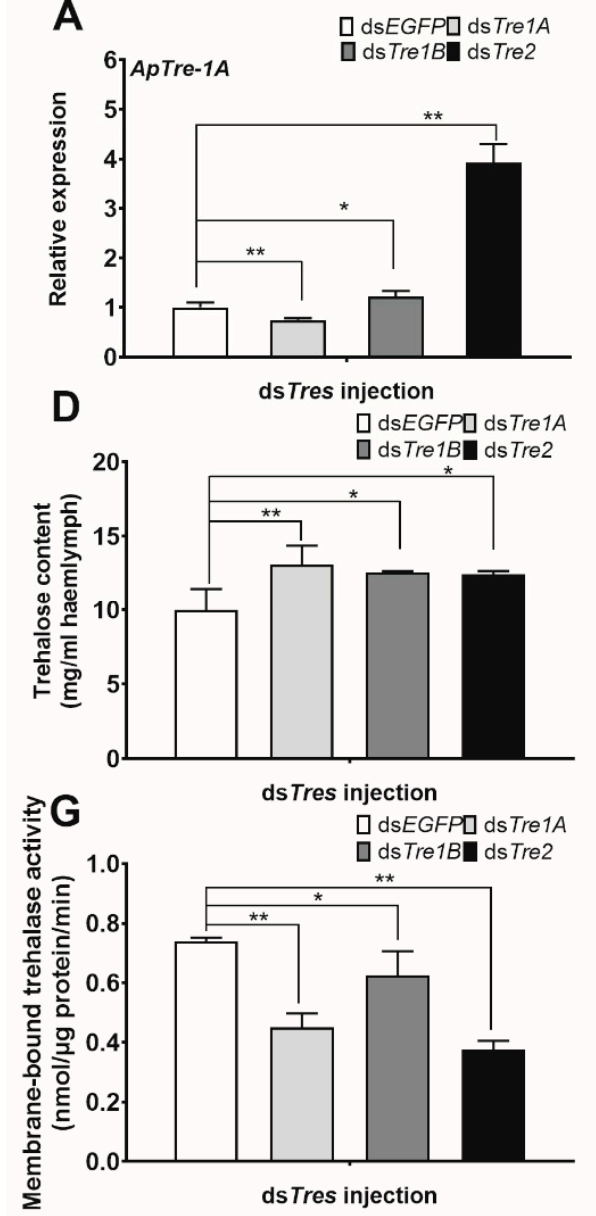

B

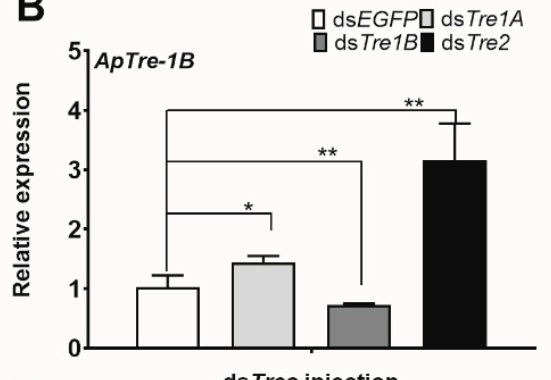

E

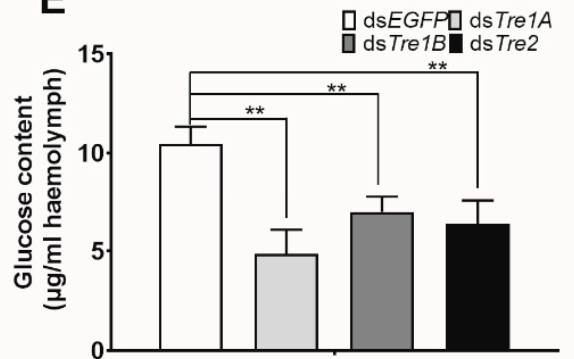

dsTres injection
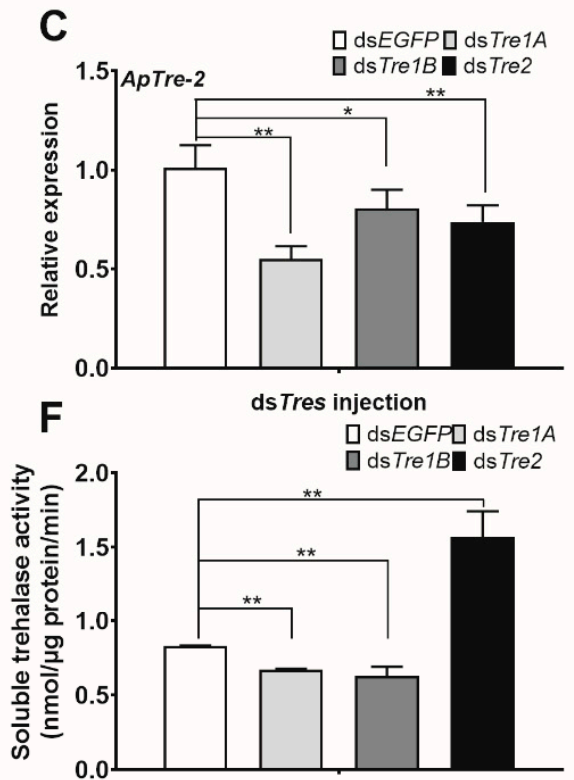

dsTres injection

Figure 6. Effects of RNAi targeting the three ApTres on trehalose catabolism in A. pernyi pupae. (A-C) Relative expression levels of ApTre-1A, ApTre-1B, and ApTre-2, respectively, at $48 \mathrm{~h}$ after RNAi targeting the three ApTres. (D,E) Changes in trehalose and glucose content, respectively, in the haemolymph at $48 \mathrm{~h}$ after RNAi targeting the three ApTres. (F,G) Changes in soluble and membrane-bound trehalase activity, respectively, at $48 \mathrm{~h}$ after RNAi targeting the three ApTres. mRNA levels were normalised to those of the reference genes actin and RP49, and the group injected with dsEGFP served as controls. Data are means of three independent experiments, and error bars are SEs. Single asterisks $(p<0.05)$ and double asterisks $(p<0.01)$ represent significant differences between the dsEGFP and dsApTres groups in the same time period.

\section{Discussion}

ILP is involved in the regulation of the metabolism, growth, reproduction, and lifespan of insects [35]. Many insect ILPs have been identified in different species, including the fruit fly D. melanogaster [6], the silkworm B. mori [36], the cotton leafworm Spodoptera littoralis [37], the beet armyworm Spodoptera exigua [38], the brown planthopper Nilaparvata lugens [39], and the mosquito Anopheles gambiae [40], as well as the locusts Locusta migratoria [41], and Schistocerca gregaria [42]. Insect ILPs share a similar structure with vertebrate insulin, and its precursors comprise a signal peptide, B chain, interconnecting C peptide, and A chain. Except for insulin growth factors, the ILP precursors are generally processed into an active form that is A- and B-chain-linked by disulphide bonds after removal of the $C$ peptide [43]. In the present study, an ILP of A. pernyi was cloned and identified based on its high amino acid sequence similarities with other known insect ILPs. In addition, the identified ILP contained a signal peptide and a B-C-A chain, which includes conserved cysteine residues and dibasic cleavage sites for the proteolytic cleavage of $C$ peptide. This finding suggests that ApILP may be activated by the removal of the $\mathrm{C}$ peptide and the formation of disulphide bridges of the B-A chain (Figure S2A). 
Many insects have been reported to possess multiple ILPs. These ILPs are at least in part expressed in a tissue-specific or stage-specific manner, which is associated with their distinct functions [43]. A specific ILP is predominantly expressed in the fat body [42,44]. In B. mori, the IGF-like peptide Bommo-IGFLP (BIGFLP) is mainly produced in the fat body during pupa-adult development, and its secretion is stimulated by the ecdysteroid 20-hydroxyecdysone (20E) [45]. Similarly, Drosophila insulin-like peptide 6 (DILP6) is expressed in the fat body during the postfeeding stage in response to ecdysteroids [46]. In this study, $A$ A ILP was also predominantly expressed in the fat body at the pupal stage (Figure S3A). Moreover, the mRNA level of ApILP in the fat body increased $~ 3.26$-fold after diapausing pupae were treated with exogenous 20E (Figure S3B); this suggests that ApILP is regulated by $20 \mathrm{E}$ at a transcriptional level, which coincides with the high expression level of ApILP during the late larval stage. Thus, it can be assumed that ApILP is a counterpart of this specific ILP, akin to BIGFLP and DILP6, and is functionally important to the A. pernyi fat body. The insect fat body, equivalent to vertebrate liver and adipose tissue, is a major site of nutrient storage and energy metabolism. Existing literature has shown that the fat body plays a central role in the integration of hormonal and nutritional signals, regulating insect development and behaviour, including pupal diapause [47]. In diapausing H. armigera pupae, the fat body can exert an effect on the brain's regulatory centre by altering the levels of sugar and tricarboxylic acid (TCA) metabolites [48]. This evidence suggests that the fat body might play an important role in diapause termination. Furthermore, this study showed that an increase in ApILP expression is associated with diapause termination in diapausing A. pernyi pupae (Figure 1C). Thus, it can be assumed that the fat-body-derived ILP of A. pernyi might play a potential role in diapause termination.

It has been reported that treatment with vertebrate insulin can lead to diapause termination via activation of the prothoracic gland in diapausing P. brassicae and Antheraea mylitta pupae $[23,49]$. Alternatively, exogenous insulin directly exerts its effects on target tissues and improves their metabolic rate, which also stimulates diapause termination [50]. In the present study, injection of bovine insulin could effectively trigger the adult emergence of diapausing A. pernyi pupae (Figure 2A). Diapause termination is related to the energy metabolism of diapausing individuals, and is characterized by an elevated respiration rate [8]. As the only enzyme that hydrolyses trehalose into glucose, trehalase regulates both energy metabolism and glucose generation via trehalose catabolism [51]; this suggests that trehalase might play an important role in diapause termination and subsequent development. The results of the present study show that changes in gene expression differ slightly between ApTre-1A, ApTre-1B, and ApTre-2 following bovine insulin injection; however, the overall expression trends of these genes increased, which may be related to the functional specificity of trehalase (Figure 2B-D). Exogenous insulin induced a significant increase in soluble and membrane-bound trehalase activity in diapausing $A$. pernyi pupae, thereby leading to a significant decline in trehalose levels in the haemolymph (Figure 2E-G). Following bovine insulin treatment, the levels of glucose in the haemolymph showed a trend of first decreasing and then increasing, which was followed by a decrease in comparison to the PBS-buffer-treated group (Figure $2 \mathrm{H}$ ); this trend was likely caused by subsequent changes in glucose levels in pupae treated with bovine insulin. The consumption of glucose was accelerated with the increased metabolic respiration of diapausing pupae, while the elevated trehalase activity promoted the hydrolysis of trehalose into glucose. This resulted in an increased glucose concentration, and a decrease in trehalase activity led to a decline in glucose levels in the haemolymph. These results indicate that vertebrate insulin elevates trehalose catabolism in diapausing A. pernyi pupae. In agreement with these results, injection of vertebrate insulin suppressed haemolymph trehalose levels in lepidopteran insects, including S. exigua and M. vitrata $[20,38]$. Thus, the obtained results suggest that vertebrate insulin could enhance trehalose catabolism and promote pupa-toadult development in diapausing A. pernyi pupae. We previously studied the action of exogenous 20E on trehalose catabolism in diapausing A. pernyi pupae [17]. Similarities exist between the effects of exogenous $20 \mathrm{E}$ and bovine insulin, according to their contribution 
to the elevated trehalose catabolism. Injections of 20E and bovine insulin both induced the upregulation of ApTre-1A and ApTre 2 which, in turn, increased the enzyme activity of soluble and membrane-bound trehalase, leading to a decline in haemolymph trehalose levels. Similar to the effects of bovine insulin and 20E on trehalose in diapausing pupae, the regulation of trehalose by juvenile hormone analogues in diapausing adults has also been reported. For example, methoprene treatment could effectively disrupt imaginal diapause and caused a marked increase in locomotory activity accompanied by a decline in trehalose activity in females of the apple blossom weevil Anthonomus pomorum [52]. This evidence indicates that the enhanced trehalose catabolism might play an important role in the diapause termination process.

Because vertebrate insulin can modulate trehalose levels, the effect of ApILP on trehalose catabolism was investigated via an RNAi experiment. The RNAi of ApILP decreased the mRNA levels of ApTre-1A and ApTre-2, which led to an increase in the trehalose content and, in turn, decreased the glucose levels (Figure 3 ). These results are consistent with a previous study that reported that the knockdown of an ILP gene (SeILP1), which is mainly expressed in the fat body, induced a significant increase in circulating trehalose levels in S. exigua [38]. It has been reported that, in D. melanogaster, trehalase exists in two forms, including cytoplasmic and putative secreted forms, and that a compensation mechanism exists between trehalase isoforms [53]. In the present study, ApTre-1B expression increased significantly after dsApILP injection, which might be a form of compensation for the decreases in ApTre- $1 A$ and ApTre-2 expression, providing the energy needed for survival (Figure $3 C$ ). It has been reported that, in A. pernyi, trehalose concentration remains at a high level during diapause maintenance, but declines when diapause is broken $[16,17]$. This change in trehalose concentration indicates that the reduction in trehalose concentration is associated with diapause termination in A. pernyi. Based on the contribution of ApILP to regulating trehalose levels, and the trend of $A p I L P$ expression during diapause termination, we speculate that $A p I L P$ is responsible for the downregulation of trehalose levels in the haemolymph of A. pernyi by increasing the expression of ApTre- $1 A$ and ApTre-2 during diapause termination.

Insulin signalling is considered a diapause regulator, as it controls developmental processes and glycolipid metabolism in insects $[5,54,55]$. A high insulin level represses FoxO activity through p-Akt and, subsequently, activates many target genes that promote insect development. In contrast, shutdown of insulin signalling represses PI3K/Akt which, in turn, increases the activity of FoxO, and leads to the generation of the diapause phenotype [56]. Accumulation of FoxO was reported in the brain of diapausing Helicoverpa armigera pupae, and overexpression of FoxO impedes larval development and extends the lifespan at the early larval phase [57]. Similarly, in diapausing Laodelphax striatellus nymphs, FoxO mRNA expression and protein levels were found to be much higher than those of non-diapausing nymphs [58]; these results corroborate the involvement of FoxO in diapause regulation. The present study showed that overexpression of $A p F o x O$ decreased the mRNA levels of ApTre-1A and ApTre-2, and suppressed the activity of soluble and membrane-bound trehalase during diapause termination; this led to an increase in trehalose levels and a decrease in glucose levels (Figure 4). In contrast, silencing $A p$ FoxO increased ApTre-1A and ApTre-2 expression, as well as the activity of soluble and membrane-bound trehalase, which accelerated the hydrolysis of trehalose in diapausing pupae (Figure 5). Consistent with the results of the present study, knockdown of LSFoxO significantly increased trehalase activity and shortened the diapause duration in L. striatellus nymphs [58]. Additionally, knockdowns of ApTre-1A and ApTre-2 both induced an increase in $A p$ Tre-1B expression (Figure 6B), suggesting that changes in ApTre- $1 B$ expression might be affected by the expression of ApTre-1A and ApTre-2 after overexpressing and silencing $A p F o x O$ (Figures $4 \mathrm{~A}$ and $5 \mathrm{~A}$ ). In summary, $\mathrm{ApFoxO}$ may modulate trehalose catabolism, thus affecting pupa-adult development in A. pernyi. 


\section{Conclusions}

In conclusion, the present study shows that an increase in ApILP expression is associated with diapause termination in A. pernyi pupae. The application of the ApILP homologous vertebrate insulin effectively stimulated diapause termination and elevated the trehalose catabolism of diapausing A. pernyi pupae. In contrast, the RNAi of ApILP suppressed trehalose hydrolysis through the downregulation of ApTre- $1 A$ and ApTre-2 expression and the reduction in the activity of soluble and membrane-bound trehalase during diapause termination. In addition, overexpression of the transcription factor $A p F o x O$ induced trehalose accumulation during diapause termination, while the silencing of $A p F o x O$ enhanced trehalose breakdown in diapausing pupae. As the main transcriptional effector of the IIS pathway, ApFoxO exhibited a contrasting effect on the trehalose catabolism to that of ApILP (Figure 7).

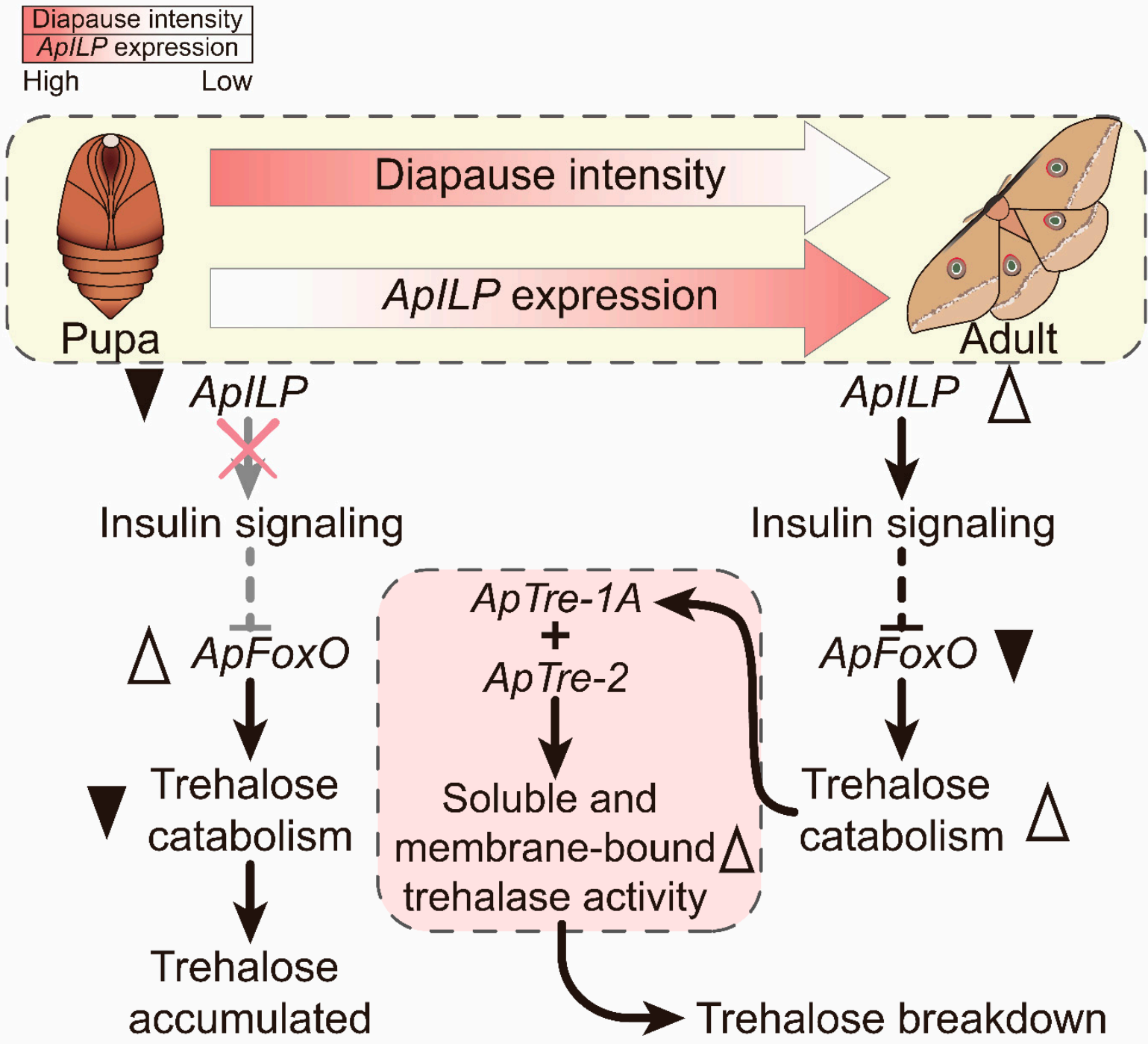

Figure 7. Schematic depiction showing that $A p I L P$ and $A p F o x O$ are involved in the regulation of the trehalose catabolism in the diapause termination process in $A$. pernyi pupae. When $A$. pernyi pupae undergo diapause, the expression of $A p I L P$ is relatively low, and insulin signalling is repressed which, in turn, stimulates an increase in $A p F o x O$ activity; then, $A p F o x O$ reduces trehalase activity, thus suppressing trehalose hydrolysis. However, the expression of ApILP increases during diapause termination, which inhibits $A p F o x O$ activity through the insulin/insulin-like growth factor signalling pathway. Subsequently, the increase in ApTre1A and ApTre2 expression triggers the activity of soluble and membrane-bound trehalase, which enhances trehalose catabolism and accelerates trehalose breakdown. Black indicates gene activation, while grey indicates deactivation. 
Supplementary Materials: The following are available online at https:/ / www.mdpi.com/article / 10.3390/insects12090784/s1: Figure S1: Phylogenetic analysis of ApILP and ApFoxO; Figure S2: Alignment analysis of ApILP and ApFoxO; Figure S3: Expression of Antheraea pernyi ApILP; Table S1: Primer sequences used in this study.

Author Contributions: Conceptualization, Y.-N.L. and W.-L.L.; methodology and sampling, Y.-N.L., X.-B.R., Z.-C.L. and W.-L.L.; investigation, Y.-N.L., X.-B.R., Z.-C.L., B.Y., Z.-J.Z. and Q.F.; software and data curation, Y.-B.L. and J.-N.Z.; writing-original draft preparation, Y.-N.L.; writing-review and editing, Y.-N.L. and W.-L.L.; project administration, Y.-N.L. and W.-L.L.; funding acquisition, Y.-B.L., J.-N.Z. and W.-L.L. All authors have read and agreed to the published version of the manuscript.

Funding: This study was supported by grants from Natural Science Foundation of China (31870793) and Natural Science Foundation of Liaoning Province of China (81165001).

Institutional Review Board Statement: Not applicable.

Informed Consent Statement: Not applicable.

Data Availability Statement: The data presented in this study can be found in the supplementary materials.

Conflicts of Interest: We declare that there is no conflict of interest in this work.

\section{References}

1. Kostál, V. Eco-physiological phases of insect diapause. J. Insect Physiol. 2006, 52, 113-127. [CrossRef] [PubMed]

2. Pullin, A.S. Diapause metabolism and changes in carbohydrates related to cryoprotection in Pieris brassicae. J. Insect Physiol. 1992, 38, 319-327. [CrossRef]

3. Denlinger, D.L. Regulation of diapause. Annu. Rev. Entomol. 2002, 47, 93-122. [CrossRef] [PubMed]

4. King, A.M.; MacRae, T.H. Insect heat shock proteins during stress and diapause. Annu. Rev. Entomol. 2015, 60, 59-75. [CrossRef] [PubMed]

5. Hahn, D.A.; Denlinger, D.L. Energetics of insect diapause. Annu. Rev. Entomol. 2011, 56, 103-121. [CrossRef] [PubMed]

6. Kubrak, O.I.; Kučerová, L.; Theopold, U.; Nylin, S.; Nässel, D.R. Characterization of Reproductive Dormancy in Male Drosophila melanogaster. Front. Physiol. 2016, 7, 572. [CrossRef]

7. Hahn, D.A.; Denlinger, D.L. Meeting the energetic demands of insect diapause: Nutrient storage and utilization. J. Insect Physiol. 2007, 53, 760-773. [CrossRef] [PubMed]

8. Dong, Y.C.; Chen, Z.Z.; Clarke, A.R.; Niu, C.Y. Changes in Energy Metabolism Trigger Pupal Diapause Transition of Bactrocera minax After 20-Hydroxyecdysone Application. Front. Physiol. 2019, 10, 1288. [CrossRef]

9. Li, Q.; Li, Y.P.; Ambühl, D.; Liu, Y.Q.; Qin, L. Nutrient composition of Chinese oak silkworm, Antheraea pernyi, a traditional edible insect in China: A review. J. Insects Food Feed. 2020, 6, 1-16. [CrossRef]

10. Williams, C.M.; Adkisson, P.L. Photoperiodic Control of Pupal Diapause in the Silkworm, Antheraea pernyi. Science 1964, $144,569$.

11. Guo, Q.; Hao, Y.J.; Li, Y.; Zhang, Y.J.; Ren, S.; Si, F.L.; Chen, B. Gene cloning, characterization and expression and enzymatic activities related to trehalose metabolism during diapause of the onion maggot Delia antiqua (Diptera: Anthomyiidae). Gene 2015, 565, 106-115. [CrossRef] [PubMed]

12. Xu, J.; Bao, B.; Zhang, Z.F.; Yi, Y.Z.; Xu, W.H. Identification of a novel gene encoding the trehalose phosphate synthase in the cotton bollworm, Helicoverpa armigera. Glycobiology 2009, 19, 250-257. [CrossRef] [PubMed]

13. Yang, F.; Chen, S.; Dai, Z.M.; Chen, D.F.; Duan, R.B.; Wang, H.L.; Jia, S.N.; Yang, W.J. Regulation of trehalase expression inhibits apoptosis in diapause cysts of Artemia. Biochem. J. 2013, 456, 185-194. [CrossRef] [PubMed]

14. Rozsypal, J.; Koštál, V.; Zahradníčková, H.; Šimek, P. Overwintering strategy and mechanisms of cold tolerance in the codling moth (Cydia pomonella). PLoS ONE 2013, 8, e61745. [CrossRef]

15. Bemani, M.; Izadi, H.; Mahdian, K.; Khani, A.; Amin Samih, M. Study on the physiology of diapause, cold hardiness and supercooling point of overwintering pupae of the pistachio fruit hull borer, Arimania comaroffi. J. Insect Physiol. 2012, 58, 897-902. [CrossRef]

16. Lu, M.X.; Yang, C.S.; Teng, X.Y.; Zhang, Y.C.; Xu, Q.M. Dynamic temperature and photoperiodic effects of carbohydrate content in the fat body and haemolymph of the Antheraea pernyi (Lepidoptera: Saturniidae) pupae. Acta Entom. Sin. 1992, 35, 1-7.

17. Li, Y.N.; Liu, Y.B.; Xie, X.Q.; Zhang, J.N.; Li, W.L. The Modulation of Trehalose Metabolism by 20-Hydroxyecdysone in Antheraea pernyi (Lepidoptera: Saturniidae) During its Diapause Termination and Post-Termination Period. J. Insect Sci. 2020, $20,18$. [CrossRef]

18. Satake, S.; Masumura, M.; Ishizaki, H.; Nagata, K.; Kataoka, H.; Suzuki, A.; Mizoguchi, A. Bombyxin, an insulin-related peptide of insects, reduces the major storage carbohydrates in the silkworm Bombyx mori. Comp. Biochem. Physiol. B Biochem. Mol. Biol. 1997, 118, 349-357. [CrossRef] 
19. Broughton, S.J.; Piper, M.D.; Ikeya, T.; Bass, T.M.; Jacobson, J.; Driege, Y.; Martinez, P.; Hafen, E.; Withers, D.J.; Leevers, S.J.; et al. Longer lifespan, altered metabolism, and stress resistance in Drosophila from ablation of cells making insulin-like ligands. Proc. Natl. Acad. Sci. USA 2005, 102, 3105-3110. [CrossRef]

20. Al Baki, M.A.; Jung, J.K.; Kim, Y. Regulation of hemolymph trehalose titers by insulin signaling in the legume pod borer, Maruca vitrata (Lepidoptera: Crambidae). Peptides 2018, 106, 28-36. [CrossRef]

21. Accili, D.; Arden, K.C. FoxOs at the crossroads of cellular metabolism, differentiation, and transformation. Cell 2004, 117, 421-426. [CrossRef]

22. Hibshman, J.D.; Doan, A.E.; Moore, B.T.; Kaplan, R.E. daf-16/FoxO promotes gluconeogenesis and trehalose synthesis during starvation to support survival. Elife 2017, 6, e30057. [CrossRef]

23. Arpagaus, M. Vertebrate insulin induces diapause termination in Pieris brassicae pupae. Rouxs Arch. Dev. Biol. 1987, 196, 527-530. [CrossRef]

24. Liu, Y.; Zhang, Q.; Denlinger, D.L. Imidazole derivative KK-42 boosts pupal diapause incidence and delays diapause termination in several insect species. J. Insect Physiol. 2015, 74, 38-44. [CrossRef] [PubMed]

25. Wang, L.M.; Zhang, B.; Ye, B.; Zhao, Z.J.; Li, S.Y.; Li, W.L.; Yue, D.M.; Fan, Q. Expression of enhanced green fluorescent protein in Antheraea pernyi pupa by using Antheraea pernyi nucleopolyhedrovirus expression vector system. Sci. Seric. 2010, 36, 792-799.

26. Ye, B.; Wang, L.M.; Zhao, Z.J.; Yue, D.M.; Zhang, H.B.; Li, S.Y.; Fan, Q. Expression of human growth hormone in Antheraea pernyi pupa using Antheraea pernyi nucleopolyhedrovirus expression vector system. Sci. Seric. 2014, 40, 0277-0283.

27. Reed, L.; Muench, H. A simple method of estimating fifty per cent endpoints. Am. J. Hyg. 1938, 27, $493-497$.

28. Livak, K.J.; Schmittgen, T.D. Analysis of relative gene expression data using real-time quantitative PCR and the 2(-Delta Delta C(T)) Method. Methods 2001, 25, 402-408. [CrossRef]

29. Nakamatsu, Y.; Tanaka, T. Correlation between concentration of hemolymph nutrients and amount of fat body consumed in lightly and heavily parasitized hosts (Pseudaletia separata). J. Insect Physiol. 2004, 50, 135-141. [CrossRef] [PubMed]

30. Tatun, N.; Singtripop, T.; Tungjitwitayakul, J.; Sakurai, S. Regulation of soluble and membrane-bound trehalase activity and expression of the enzyme in the larval midgut of the bamboo borer Omphisa fuscidentalis. Insect Biochem. Mol. Biol. 2008, 38, 788-795. [CrossRef] [PubMed]

31. Sim, C.; Denlinger, D.L. Insulin signaling and the regulation of insect diapause. Front. Physiol. 2013, 4, 189. [CrossRef]

32. Tatar, M.; Kopelman, A.; Epstein, D.; Tu, M.P.; Yin, C.M.; Garofalo, R.S. A mutant Drosophila insulin receptor homolog that extends life-span and impairs neuroendocrine function. Science 2001, 292, 107-110. [CrossRef]

33. Williams, K.D.; Busto, M.; Suster, M.L.; So, A.K.; Ben-Shahar, Y.; Leevers, S.J.; Sokolowski, M.B. Natural variation in Drosophila melanogaster diapause due to the insulin-regulated PI3-kinase. Proc. Natl. Acad. Sci. USA 2006, 103, 15911-15915. [CrossRef]

34. Sim, C.; Denlinger, D.L. A shut-down in expression of an insulin-like peptide, ILP-1, halts ovarian maturation during the overwintering diapause of the mosquito Culex pipiens. Insect Mol. Biol. 2009, 18, 325-332. [CrossRef]

35. Steele, J.E. Control. of Metabolic Processes. In Comprehensive Insect Physiology, Biochemistry and Pharmacology; Pergamon Press: New York, NY, USA, 1985; pp. 99-145.

36. Yoshida, I.; Moto, K.; Sakurai, S.; Iwami, M. A novel member of the bombyxin gene family: Structure and expression of bombyxin G1 gene, an insulin-related peptide gene of the silkmoth Bombyx mori. Dev. Genes Evol. 1998, 208, 407-410. [CrossRef]

37. Van de Velde, S.; Badisco, L.; Claeys, I.; Verleyen, P.; Chen, X.; Vanden Bosch, L.; Vanden Broeck, J.; Smagghe, G. Insulin-like peptides in Spodoptera littoralis (Lepidoptera): Detection, localization and identification. Gen. Comp. Endocrinol. 2007, 153, 72-79. [CrossRef]

38. Kim, Y.; Hong, Y. Regulation of hemolymph trehalose level by an insulin-like peptide through diel feeding rhythm of the beet armyworm, Spodoptera exigua. Peptides 2015, 68, 91-98. [CrossRef] [PubMed]

39. Xue, W.H.; Liu, Y.L.; Jiang, Y.Q.; He, S.F.; Wang, Q.Q.; Yang, Z.N.; Xu, H.J. Molecular characterization of insulin-like peptides in the brown planthopper, Nilaparvata lugens (Hemiptera: Delphacidae). Insect Mol. Biol. 2020, 29, 309-319. [CrossRef]

40. Krieger, M.J.; Jahan, N.; Riehle, M.A.; Cao, C.; Brown, M.R. Molecular characterization of insulin-like peptide genes and their expression in the African malaria mosquito, Anopheles gambiae. Insect Mol. Biol. 2004, 13, 305-315. [CrossRef] [PubMed]

41. Lagueux, M.; Lwoff, L.; Meister, M.; Goltzené, F.; Hoffmann, J.A. cDNAs from neurosecretory cells of brains of Locusta migratoria (Insecta, Orthoptera) encoding a novel member of the superfamily of insulins. Eur. J. Biochem. 1990, 187, 249-254. [CrossRef] [PubMed]

42. Badisco, L.; Claeys, I.; Van Hiel, M.; Clynen, E.; Huybrechts, J.; Vandersmissen, T.; Van Soest, S.; Vanden Bosch, L.; Simonet, G.; Vanden Broeck, J. Purification and characterization of an insulin-related peptide in the desert locust, Schistocerca gregaria: Immunolocalization, cDNA cloning, transcript profiling and interaction with neuroparsin. J. Mol. Endocrinol. 2008, 40, 137-150. [CrossRef]

43. Nässel, D.R.; Vanden Broeck, J. Insulin/IGF signaling in Drosophila and other insects: Factors that regulate production, release and post-release action of the insulin-like peptides. Cell Mol. Life Sci. 2016, 73, 271-290. [CrossRef] [PubMed]

44. Nilsen, K.A.; Ihle, K.E.; Frederick, K.; Fondrk, M.K.; Smedal, B.; Hartfelder, K.; Amdam, G.V. Insulin-like peptide genes in honey bee fat body respond differently to manipulation of social behavioral physiology. J. Exp. Biol. 2011, 214, 1488-1497. [CrossRef] [PubMed]

45. Okamoto, N.; Yamanaka, N.; Satake, H.; Saegusa, H.; Kataoka, H.; Mizoguchi, A. An ecdysteroid-inducible insulin-like growth factor-like peptide regulates adult development of the silkmoth Bombyx mori. FEBS J. 2009, 276, 1221-1232. [CrossRef] 
46. Okamoto, N.; Yamanaka, N.; Yagi, Y.; Nishida, Y.; Kataoka, H.; O'Connor, M.B.; Mizoguchi, A. A fat body-derived IGF-like peptide regulates postfeeding growth in Drosophila. Dev. Cell 2009, 17, 885-891. [CrossRef]

47. Li, S.; Yu, X.; Feng, Q. Fat Body Biology in the Last Decade. Annu. Rev. Entomol. 2019, 64, 315-333. [CrossRef]

48. Xu, W.H.; Lu, Y.X.; Denlinger, D.L. Cross-talk between the fat body and brain regulates insect developmental arrest. Proc. Natl. Acad. Sci. USA 2012, 109, 14687-14692. [CrossRef]

49. Sinha, A.K.; Chakraborty, D.; Chaudhuri, A. Insulin-induced effect on diapausing pupae of tropical tasar silkworm, Antheraea mylitta Drury (Lepidoptera: Saturniidae). Insect Sci. Appl. 1993, 14, 707-712. [CrossRef]

50. Denlinger, D.L. Hormonal Control. of Diapause. In Comprehensive Insect Physiology, Biochemistry and Pharmacology; Pergamon Press: New York, NY, USA, 1985; pp. 353-412.

51. Shukla, E.; Thorat, L.J.; Nath, B.B.; Gaikwad, S.M. Insect trehalase: Physiological significance and potential applications. Glycobiology 2015, 25, 357-367. [CrossRef] [PubMed]

52. Zdarek, J.; Ctvrtecka, R.; Hovorka, O.; Kostal, V. Activation of gonads and disruption of imaginal diapause in the apple blossom weevil, Anthonomus pomorum (Coleoptera: Curculionidae), with juvenoids in laboratory and field trials. Eur. J. Entomol. 2000, 97, 25-31. [CrossRef]

53. Yoshida, M.; Matsuda, H.; Kubo, H.; Nishimura, T. Molecular characterization of Tps1 and Treh genes in Drosophila and their role in body water homeostasis. Sci. Rep. 2016, 6, 30582. [CrossRef]

54. Sim, C.; Denlinger, D.L. Insulin signaling and FOXO regulate the overwintering diapause of the mosquito Culex pipiens. Proc. Natl. Acad. Sci. USA 2008, 105, 6777-6781. [CrossRef] [PubMed]

55. Denlinger, D.L.; Yocum, G.D.; Rinehart, J.P. Hormonal Control. of Diapause. In Comprehensive Molecular Insect Science; Elsevier: Amsterdam, The Netherlands, 2005; pp. 615-650.

56. Sim, C.; Kang, D.S.; Kim, S.; Bai, X.; Denlinger, D.L. Identification of FOXO targets that generate diverse features of the diapause phenotype in the mosquito Culex pipiens. Proc. Natl. Acad. Sci. USA 2015, 112, 3811-3816. [CrossRef] [PubMed]

57. Zhang, X.S.; Wang, T.; Lin, X.W.; Denlinger, D.L.; Xu, W.H. Reactive oxygen species extend insect life span using components of the insulin-signaling pathway. Proc. Natl. Acad. Sci. USA 2017, 114, E7832-E7840. [CrossRef] [PubMed]

58. Yin, Z.J.; Dong, X.L.; Kang, K.; Chen, H.; Dai, X.Y.; Wu, G.A.; Zheng, L.; Yu, Y.; Zhai, Y.F. FoxO transcription factor regulate hormone mediated signaling on nymphal diapause. Front. Physiol. 2018, 9, 1654. [CrossRef] 